\title{
Les ouvrages de vulgarisation scientifique au Canada français
}

L'influence du frère Marie-Victorin sur la littérature pour la jeunesse

\section{Lucie Guillemette}

\section{(2) OpenEdition}

\section{Journals}

Édition électronique

URL : http://journals.openedition.org/abpo/1361

DOI : 10.4000/abpo.1361

ISBN : 978-2-7535-1492-8

ISSN : 2108-6443

\section{Éditeur}

Presses universitaires de Rennes

\section{Édition imprimée}

Date de publication : 20 décembre 2003

Pagination : 235-246

ISBN : 978-2-86847-933-4

ISSN : 0399-0826

\section{Référence électronique}

Lucie Guillemette, "Les ouvrages de vulgarisation scientifique au Canada français », Annales de Bretagne et des Pays de l'Ouest [En ligne], 110-4 | 2003, mis en ligne le 20 décembre 2005, consulté le 01 mai 2019. URL : http://journals.openedition.org/abpo/1361 ; DOI : 10.4000/abpo.1361 


\title{
Les ouvrages de vulgarisation scientifique au Canada français
}

\author{
L'influence du frère Marie-Victorin \\ sur la littérature pour la jeunesse
}

\author{
Lucie GuILLEMETTE \\ Professeur de Littérature moderne \\ Université du Québec à Trois-Rivières \\ Centre interuniversitaire d'études québécoises \\ Laboratoire des littératures françaises d'Amérique pour la jeunesse
}

Nombreux et variés sont les savoirs qui se greffent sur les œuvres québécoises pour la jeunesse depuis les premières parutions jusqu'à aujourd'hui. Née dans les années 1920, la littérature destinée à un jeune public témoigne des multiples activités à caractère pédagogique développées au fil du siècle pour instruire et divertir les enfants. Compte tenu d'un destinataire qui, par définition, est surdéterminé, les textes rendent compte également des efforts de vulgarisation mis en œuvre pour rendre le propos accessible, surtout lorsqu'il comporte des éléments à teneur scientifique. Il importe de mentionner que les premiers écrits à l'intention de la jeunesse sont des contes historiques publiés sous l'égide de la Société Saint-JeanBaptiste de 1919 à 1923. Organisme dont la mission consiste encore aujourd'hui à valoriser la culture et la langue françaises au Québec, la Société SaintJean-Baptiste souhaitait à l'époque promouvoir le patriotisme dès le jeune âge et intéresser les enfants à l'histoire nationale : "Axés essentiellement sur les héros et les hauts faits de la Nouvelle-France, les Contes historiques sont publiés par des écrivains chevronnés ${ }^{1}$. " Si les premiers récits pour la jeunesse sollicitent un savoir historique, ils font appel également à d'autres disciplines scientifiques. Plus précisément, notre programme de recherche consiste à identifier les connaissances encyclopédiques qui circulent dans des textes signés par des femmes et destinés à un jeune lectorat, de façon à décrire et à analyser les passages mettant en lumière les liens significatifs à d'autres champs disciplinaires, qu'ils relèvent des sciences pures et appli-

1. LEPAGE, Françoise, Histoire de la littérature pour la jeunesse. Québec et francophonies du Canada suivie d'un Dictionnaire des auteurs et des illustrateurs, Orléans (Canada), Les Éditions David, 2000, p. 110. 
quées comme les mathématiques, la physique, les sciences naturelles, ou qu'ils sollicitent des connaissances d'ordre philosophique et historique. L'influence des écrits du frère Marie-Victorin sur des œuvres littéraires pour la jeunesse retiendra tout particulièrement notre attention dans la mesure où les travaux de ce dernier dans le domaine des sciences naturelles ont inspiré nombre d'écrivains durant les années 1930 et 1940. Dans le cadre de cette étude, il importe d'examiner d'abord les écrits du frère Marie-Victorin afin de décrire son influence à la fois dans le milieu scientifique et dans le champ littéraire. Par la suite, il s'agira de cerner la fonction de vulgarisation des textes littéraires pour la jeunesse dont le code est dicté en grande partie par l'œuvre de l'homme de science.

\section{Le frère Marie-Victorin et la percée des sciences au Canada français}

Botaniste de formation, le frère Marie-Victorin des Écoles chrétiennes ${ }^{2}$, né Conrad Kirouac en 1885 et décédé en 1944, a entamé une carrière dans l'enseignement primaire avant de devenir professeur de botanique à la faculté des sciences de l'Université de Montréal en 1919. Il a assumé également les fonctions de président de la Société canadienne d'histoire naturelle de 1925 à 1940. Notons qu'il est le fondateur du Jardin botanique de Montréal. Dans un article paru en 1942, Georges Maheux, un spécialiste d'entomologie, décrit l'homme de science " comme le chef incontesté des biologistes du Canada français ${ }^{3}$ ". Nul doute que les travaux du frère Marie-Victorin ont contribué à l'essor des sciences naturelles dans le monde de l'enseignement à une époque où l'on ne croyait guère à l'influence formatrice des sciences au Canada français, les sciences naturelles étant reléguées au statut de "petites sciences". Selon toute vraisemblance, l'expression, qui désignait les sciences de la nature (astronomie, biologie, botanique, géologie, zoologie, etc.), serait de $\mathrm{M}^{\mathrm{gr}}$ Joseph-Clovis-Kemner Laflamme, le premier véritable géologue du Canada français, qui fut l'une des figures marquantes du milieu scientifique à la fin du $19^{\mathrm{e}}$ siècle. "[ $\left.\mathrm{M}^{\mathrm{gr}} \mathrm{Laflamme}\right]$ entendait désigner par ce vocable ["petites sciences"] des spécialités nouvelles ${ }^{4}$ ", encore au stade des balbutiements. Or, à titre de botaniste, le frère Marie-Victorin voulait que les sciences naturelles acquièrent leurs lettres de noblesse ou qu'elles deviennent, à tout le moins, partie intégrante de la culture générale. Et il n'était pas le seul. Afin de montrer la place restreinte qu'occupaient les sciences expérimentales dans les programmes d'enseignement au cours des années vingt, Henri Prat insiste sur " la valeur éducative des sciences de la nature dans la formation de l'esprit " au sein d'un article paru en $1929^{5}$. L'auteur, un agrégé

2. Le frère Marie-Victorin est un frère des écoles chrétiennes, d'où l'abréviation f.e.c. parfois utilisée après le nom.

3. MAHEUX, Georges, "Frère Marie-Victorin. Le savant. Son œuvre ", Regards, vol. 3, n 89, mai-juin 1942, p. 344.

4. AUDET, Louis-Philippe, Le Frère Marie-Victorin éducateur. Ses idées pédagogiques, Québec, Éditions de l'Érable, 1942, note 23a, p. 47.

5. PRAT, Henri, "Valeur éducative des sciences de la nature ", Revue trimestrielle canadienne, vol. $15, \mathrm{n}^{\circ} 57,1929$, p. 54-66. 
de l'Université française devenu professeur de biologie à l'Université de Montréal, affirme que les sciences expérimentales « nous habituent à manier divers instruments : l'observation, l'expérimentation, les hypothèses de travail, les hypothèses explicatives [...] " et [qu'elles] " procurent ainsi un support matériel aux qualités d'exposition et de raisonnement façonnées dans les cours de lettres. " Prônant l'introduction des matières scientifiques dans les programmes d'enseignement, l'auteur prétend que l'œuvre de Louis Pasteur à la fin du 19e siècle en France a " fait tomber bien des préventions contre les applications des sciences biologiques " et a contribué à bien des égards à la refonte du système d'enseignement ayant conduit à la réforme de 1902 dans les lycées. Dans la même foulée, Maheux souligne qu'au début du siècle, " on portait comme une décoration son ignorance de tout ce qui constitue le milieu physique ${ }^{6}$ ". Au Canada français, explique-t-il, on faisait fi du réel car l'éducation était fermée au Livre de la nature : " Notre cas était d'autant plus inexplicable que le Québec possède des trésors d'une richesse incroyable : forêts, mines, sol, houille blanche. " Alors que s'affrontent les tenants d'une culture classique et les promoteurs des sciences dans les programmes d'enseignement de tout niveau, on publie durant les années 1930 et 1940 des ouvrages pour la jeunesse de plus en plus réceptifs au discours scientifique.

\section{L'œuvre du frère Marie-Victorin : la rencontre des sciences et des lettres}

C'est dans un contexte réfractaire à la culture scientifique que MarieVictorin publie en 1919 les Récits laurentiens et, par la suite, en 1920, les Croquis laurentiens ${ }^{7}$. Il s'agit là d'ouvrages que les jeunes parcourent au moment de leur parution bien qu'on ne leur destine pas spécifiquement. De fait, la critique s'accorde pour dire que ces textes, au même titre que les Propos canadiens de l'abbé Camille Roy ${ }^{8}$, visent davantage un public général que des enfants et des adolescents. Louise Lemieux parle pour sa part d'une littérature spontanée - qu'elle oppose à la littérature intentionnelle - où le terroir est à l'honneur :

" Beaucoup plus tard, ces ouvrages écrits pour les adultes et non pour les jeunes, s'inséreront dans des collections qui leur sont destinées; dans ces collections, on trouve soit la version intégrale, soit un texte adapté aux jeunes, de la version originale ${ }^{9}$."

Force est de reconnaître néanmoins que le recueil de nouvelles qui compose les Récits laurentiens demeure un texte bien adapté à un jeune lecto-

6. MAHEuX, Georges, art. cité, p. 338.

7. MARIE-VICTORIN, frère, F.E.C (frère des écoles chrétiennes), Récits laurentiens, préface d'Albert FERLAND, Montréal, Frères des écoles chrétiennes, 1919; Croquis laurentiens, préface d'Ernest BiLODEAU, Montréal, Frères des écoles chrétiennes, 1920.

8. Roy, Camille, Propos canadiens, Québec, Action sociale, 1912.

9. LEMIEUX, Louise, Pleins feux sur la littérature de jeunesse au Canada français, Montréal, Leméac, 1972, p. 24. 
rat en vertu d'une narration linéaire, d'un vocabulaire concis et accessible, d'illustrations qui brillent par leur simplicité. On remarque que tous les canadianismes sont en italique dans le but de mettre en relief l'espace du discours, d'une part, et de faciliter la lecture à un public plus large, d'autre part. Françoise Lepage dans son Histoire de la littérature pour la jeunesse affirme que " de 1870 à 1920, on constate une superposition quasi totale de la littérature de jeunesse et de la littérature populaire, comme en témoigne l'aspect matériel du livre. Abondamment illustrées, certaines publications pour adultes se confondent avec des livres pour la jeunesse ${ }^{10}$ ". À travers les Récits laurentiens, le frère Marie-Victorin expose au lecteur les richesses naturelles du territoire de la vallée du Saint-Laurent au fil de six nouvelles qui se présentent à la fois comme des textes autobiographiques inspirés de l'enfance de l'auteur et des courts récits qui mettent en scène des personnages historiques ou fictifs. Dans la préface de l'édition de 1919, Albert Ferland indique la pertinence de l'ouvrage auprès de la jeunesse qui y découvrira des paysages insoupçonnés : " À la jeunesse, sensible et avide du sens des choses, ces Récits laurentiens, apportent une fraîche révélation. » Comme le souligne Ferland, « la Terre canadienne, vue à travers la vision plus lumineuse du savant, [...] lui dévoile le sens de ses lignes géologiques ". Il poursuit en affirmant que « le frère Marie-Victorin va piller l'ornement des paysages pour en parer sa prose, et partout de sa part c'est un salut intéressé au jonc fleuri, à l'aubépine, à la vergette, comme au lis d'eau, à la sélaginelle, à la verge d'or ${ }^{11}$ ". Les Récits laurentiens marquent ainsi l'aube d'une littérature dite de vulgarisation scientifique dans la mesure où l'auteur cherche à faire découvrir au lecteur le monde vivant de la nature qui se déploie au cœur du territoire laurentien, tout en s'assurant que le propos demeure accessible, clair et vivant.

Un discours consacré à la nature posée comme un objet d'étude scande l'ensemble des textes constituant les Récits laurentiens. Dans la nouvelle qui inaugure le recueil, la disparition d'un orme gigantesque emporte le souvenir des ancêtres et entraîne pour ainsi dire la mort de son propriétaire. L'intrigue de "La corvée des Hamel " se tisse en effet autour de l'existence de Siméon Hamel, le grand-oncle du narrateur. La vie de Siméon apparaît si étroitement liée à celle de l'arbre, bien des fois centenaire, que l'homme ne pourra survivre à sa perte. C'est par ailleurs autour d'un rosier que s'articule le récit d'une légende issue de la région de l'Ancienne-Lorette, située à proximité de la ville de Québec. La légende est cette fois reproduite par la grand-mère du narrateur dans " Le rosier de la vierge ». Sous la plume du naturaliste, le rosier se transforme en un véritable protagoniste de l'action :

" Tous les automnes, le rosier livrait une à une et comme à regret ses folioles jaunies aux vents froids, puis, courageux, faisait tête aux rafales terribles accourues des Laurentides, fouettait le mur glacé, usait son écorce et ses épines aux aspérités de la pierre ${ }^{12}$."

10. LEPAGE, Françoise, op. cit., p. 51.

11. Dans la préface à MARIE-VICTORIN, Récits laurentiens, op. cit., p. 13, 11 et 7.

12. MARIE-VICTORIN, Récits laurentiens, op. cit., p. 42. 
Une autre nouvelle intitulée " Charles Roux " met en scène un personnage qui, toujours tapi dans un coin pour lire, est tourné en dérision par le jeune narrateur et ses amis. Alors qu'il explore en catimini le coffre où Charles range ses livres, le gamin met la main sur des productions hétéroclites : "Les petits Berquin ${ }^{13}$ se serraient quatre à quatre contre un dictionnaire latin; il y avait surtout une vieille édition des Études de la nature de cet aimable Bernardin de Saint-Pierre ${ }^{14}$. " Autant les ouvrages pour la jeunesse importés d'Europe, si l'on songe aux histoires d'Arnaud Berquin, autant les traités de philosophie naturelle, passionnent le jeune Conrad. Les textes à saveur autobiographique attestent également de l'éveil du gamin à la connaissance livresque. Il faut dire que déjà au $19^{\mathrm{e}}$ siècle, entre 1815 et 1819 , Le Buffon des enfants ${ }^{15}$ et le Cabinet du petit naturaliste de madame Dufrénoy ${ }^{16}$ figurent parmi les bibliothèques privées de Montréal et Québec puisqu'on importe de France de nombreux ouvrages de vulgarisation scientifique. Si les Récits laurentiens s'avèrent un vibrant hommage à la nature destiné d'abord à la jeunesse, le livre se veut également un hymne à la patrie canadienne-française et à son histoire. Une nouvelle, qui met en scène Lord Durham chargé de rédiger le désormais célèbre rapport qui a présidé à l'Acte d'Union du Bas-Canada et du Haut-Canada en 1840, renvoie, au niveau de l'atmosphère, au courant romantique qui fait de la nature le reflet de l'âme. À preuve, le Britannique se remémore " les périodes harmonieuses de Chateaubriand chantant la nuit dans les déserts du Nouveau Monde ${ }^{17}$ ", pendant qu'il observe le décor de la ville de Québec. Le gouverneur s'initie, par l'entremise d'une fille de service, qui s'avère la fille d'un Patriote et la protégée des sœurs Ursulines, à la légende de Marguerite de Verchères. En reconstituant les aventures de la jeune fille de quatorze ans qui aurait repoussé bravement les Iroquois, Thérèse veut signifier à Durham que le Canada français possède une histoire, contrairement à ce que peut penser le Britannique. Si Lionel Groulx fait de la connaissance de l'histoire le fondement même de l'éducation nationale, Marie-Victorin confère à la nature des vertus susceptibles d'as-

13. Arnaud Berquin est un écrivain français (1747-1791) qui a composé des œuvres pour la jeunesse dont L'ami des enfants (24 vol., 1782-1783), L'ami des adolescents et Le livre de famille (1784-1791). Comme ces histoires ont une visée moralisatrice, elles ont donné naissance au terme, quelque peu péjoratif, de berquinades. "L'ami des enfants est une publication mensuelle de près de [150] pages, parue de décembre 1782 à décembre 1783 " (LEPAGE, Françoise, op. cit., p. 42)

14. MARIE-VICTORIN, Récits laurentiens, ouvr. cité, p. 106. Bernardin de Saint-Pierre (17371814) a fait paraître Paul et Virginie dans le dernier volume des Études de la nature (1788). Il s'agit d'une œuvre qui présente un traité de philosophie naturelle dont les parties descriptives évoquent le monde des sensations et des couleurs. Roman idyllique qui met de l'avant les idées de Jean-Jacques Rousseau, Paul et Virginie illustre les thèses présentées dans les Études.

15. BuFFon, Georges Louis Leclerc, Le Buffon des enfants ou petite histoire naturelle des quadrupèdes, des oiseaux, des poissons, des amphibies, des insectes, etc., Paris, Belin et Le Prieur, 1809.

16. Adélaïde Dufrénoy est une écrivaine française (1765-1825).

17. MARIE-VICTORIN, Récits laurentiens, op. cit., p. 191. 
sainir la race, comme il le mentionnera dans la préface d'un ouvrage destiné à la jeunesse quelques années plus tard.

\section{Marie-Victorin : quand le savant devient critique littéraire}

Prolifique, le botaniste a rédigé plusieurs articles au fil de sa carrière. Un texte publié dans le quotidien Le Devoir en novembre 1932 a suscité la controverse. Au cœur de l'exposé, Marie-Victorin s'en prend à la terminologie à laquelle ont recours des poètes figurant parmi les plus illustres du Canada français au $19^{\mathrm{e}}$ siècle pour décrire la nature. On peut supposer que le texte du frère Marie-Victorin a eu une incidence sur les écrivains pour la jeunesse, soucieux de vulgariser correctement un discours consacré aux sciences naturelles. L'homme de sciences relève en effet de nombreuses erreurs inscrites au sein de la poésie de Louis Fréchette et de William Chapman ${ }^{18}$, laquelle, selon lui, " ne reflète pas la vraie couleur de la nature laurentienne ${ }^{19}$ ". Attentif aux imprécisions, Marie-Victorin signale les méprises scientifiques qui jalonnent Les fleurs boréales (1880) de Louis Fréchette. Il montre notamment que l'emploi du mot " ajonc" au sein des vers " Plongeant dans les ajoncs et algues verdâtres " est erroné puisque l'ajonc est une légumineuse européenne inexistante en Amérique. Selon l'auteur, Fréchette a résolument confondu " jonc " et " ajonc ". Quant à Chapman, il se fourvoie en employant l'expression " lis immaculé " au sein du poème "Renouveau ". À la lecture des vers " Au champ flotte l'odeur du lis immaculé ", Marie-Victorin réplique que le lis immaculé ne croît que dans les jardins et les serres tandis que le lis du Canada auquel se réfère Chapman a des fleurs inodores, orangées et maculées de noir. Plus encore, l'auteur expose les erreurs qui ont subsisté dans un quatrain où Chapman tente de décrire un lac des Laurentides. L'homme de science y prélève le terme " pétrel " qui, désignant un oiseau de l'océan, témoigne d'une méconnaissance de l'habitat des lieux évoqué dans le poème. Que dire des glaïeuls que l'on peut certes cultiver mais qui ne peuvent composer un tableau distinctif d'un lac en milieu sauvage. On aura compris que pareille critique avait pour but de valoriser la culture scientifique perçue par le botaniste comme indispensable aux hommes de lettres.

18. Louis Fréchette est un " écrivain canadien d'expression française (1839-1908) ". Il est l'auteur du recueil poétique La voix d'un exilé (1868) où il manifeste son talent de pamphlétaire. Il présente dans La légende d'un peuple (1887) " de grandioses descriptions de la nature nord-américaine et des évocations épiques de l'histoire du Canada. " Le Petit Robert des noms propres, Paris, Dictionnaires Le Robert, 1994, p. 780. Quant à William Chapman (1850-1917), il occupe plusieurs emplois avant de devenir poète. En 1890 paraît Les feuilles d'érable qui constitue l'un des plus imposants recueils de poésie du $19^{\mathrm{e}}$ siècle canadien-français. L'auteur y aborde le thème religieux et le sentiment de la nature.

19. MARIE-Victorin, "La couleur locale dans la littérature canadienne ", Le Devoir, 9 novembre 1932 , p. 4. 


\section{Le frère Marie-Victorin et la légitimation de l'œuvre}

La publication de l'ouvrage La flore laurentienne ${ }^{20}$ en 1935 consacre les efforts de Marie-Victorin au titre de botaniste. Il s'agit d'un manuel de base qui se pose, selon les propos de l'auteur au sein de la préface, comme « un recensement des plantes croissant sans culture dans la province de Québec, le Livre d'or de nos richesses végétales naturelles ». Ses recherches sur la phytogéographie et l'écologie de la flore du Québec lui attirent la reconnaissance du milieu scientifique. Dédié à la jeunesse, le livre salue l'existence des Cercles des jeunes naturalistes qui furent fondés en 1931 par le frère Adrien Rivard des Écoles chrétiennes : " Je dédie ce livre à la jeunesse nouvelle de mon pays, et particulièrement aux dix mille jeunes gens et jeunes filles qui forment la pacifique armée des Cercles des jeunes naturalistes ${ }^{21}$. " Toujours est-il que l'on ne peut négliger la question des sciences et les efforts consentis à les rendre accessibles à la jeune population, lorsqu'on aborde les œuvres littéraires pour la jeunesse publiées à la suite de la parution des textes majeurs du frère Marie-Victorin.

\section{Le frère Marie-Victorin et les textes pour la jeunesse des femmes}

Des ouvrages de vulgarisation à l'intention des jeunes rendent compte de l'incidence prépondérante des travaux du botaniste durant les années 1930 et 1940. De fait, des femmes ont signé des textes participant de l'effort de vulgarisation des sciences naturelles durant cette période. Que l'on songe à Odette Oligny qui fait paraître Nos animaux domestiques ${ }^{22}$ au début des années 1930. D'autres écrivaines se distinguent en publiant des textes de vulgarisation scientifique, qui s'inscrivent dans le prolongement des travaux du frère Marie-Victorin. Alice Duchesnay s'illustre tout particulièrement avec la parution de deux ouvrages : "Préfacé par le frère Marie-Victorin, son livre sur les oiseaux ${ }^{23}$ demeure encore aujourd'hui un classique dans le domaine ${ }^{24}$. " L'homme de science souhaite d'ailleurs dans sa préface que le livre de Duchesnay " fasse sa part dans l'œuvre d'assainissement de la race par le retour à la nature ". Dans un second ouvrage intitulé Mon bestiaire familier ${ }^{25}$, Duchesnay poursuit son activité d'écriture alors que son époux, Elzéar Juchereau-Duchesnay, lui-même auteur d'un livre sur la faune reconnu par les maîtres, continue de l'initier à l'histoire naturelle. Proche collaboratrice de Marie-Victorin, Marcelle Gauvreau est l'auteur de Plantes curieuses de mon pays ${ }^{26}$ qui présente à l'intention des

20. MARIE-VICTORIN, La Flore laurentienne, Montréal, Les Presses de l'Université de Montréal, 1964 [1935].

21. MARIE-VICTORIN, La Flore laurentienne, op. cit., p. 11.

22. OlignY, Odette, Nos animaux domestiques, 12 vol., Montréal, Albert Lévesque, 1933.

23. DuCHESNAY, Alice, Oiseaux de mon pays, préface du frère MARIE-VICTORIN, Montréal, Centre de psychologie et de pédagogie, 1970 [1943].

24. LEPAGE, Françoise, op. cit., p. 643.

25. DuCHESNAY, Alice, Mon bestiaire familier, 1944.

26. GaUvReau, Marcelle, Plantes curieuses de mon pays, préface du frère Marie-VICTORIN Montréal, Fides, 1943. 
enfants des rudiments de la flore spontanée du pays. Notons que Marcelle Gauvreau a collaboré à un ouvrage portant sur "les idées pédagogiques " du frère Marie-Victorin ${ }^{27}$ et qu'elle est l'auteur d'un livre consacré à la contribution du botaniste à une société savante canadienne-française ${ }^{28}$.

Écrivaine prolifique, Maxine signe pour sa part Les trois fées au bois d'épinettes ${ }^{29}$ en 1936. Maxine est le pseudonyme de Madame Alexandre Taschereau-Fortier dont la production pour la jeunesse, loin d'être ponctuelle et isolée, s'échelonne sur plus de vingt ans, soit de 1926 à 1947. Fidèle à l'enseignement de l'historien François-Xavier Garneau livré à travers son Histoire du Canada parue de 1845 à 1848, la romancière a transposé au sein de ces fictions ce que l'on reconnaissait comme vertus à la nation canadienne-française. Les trois fées du bois d'épinette dont le sous-titre est $L a$ nature, ses règnes, ses merveilles est une fantaisie documentaire, évocatrice d'un discours factuel sur les sciences naturelles, au cœur d'une fiction destinée aux enfants. Résumons l'histoire. De jeunes citadins sont ravis de se retrouver à la campagne où ils s'adonnent à diverses activités. Trois fées les interpellent et emmènent les jeunes protagonistes visiter des lieux où s'incarne un règne distinct de la nature. On aura compris que les fées représentent chacun des règnes : la fée Vigueur est associée au règne animal, la fée Croissance au règne végétal et la fée Richesse au règne minéral. D'entrée de jeu, l'éditeur Albert Lévesque souligne l'originalité de l'œuvre de Maxine, bien que le livre ait succédé à la publication, la même année, de $L ' A B C d u$ petit naturaliste canadien rédigé par Harry Bernard ${ }^{30}$. Lévesque explique que le manuscrit de Maxine avait été déposé bien avant : "S'il y a degré de parenté, c'est plutôt que L'ABC du petit naturaliste est fille de l'inspiration de Maxine. "En avant-propos, l'auteure elle-même présente le texte " comme une simple nomenclature de certains spécimens des trois règnes de la nature avec quelques mots à la portée des tout-petits ${ }^{31}$ ". À la lumière de l'énoncé, se cristallisent les composantes d'une œuvre dont le destinataire est surdéterminé. Tel le précise l'auteure, le texte qui s'adresse à des enfants doit conserver une portée didactique du début à la fin du récit pour maintenir l'intérêt. Le juge Laetare Roy confère à la fable le même caractère intentionnel que traduisent avec brio les qualités pédagogiques du texte, comme le traduit sa préface : " [L'auteur] crée trois enfants de goûts différents qui pénètrent dans ce domaine sacré où la nature sous sa triple forme, animale, végétale et minérale, se fait connaître à eux, les instruit en les amusant ${ }^{32}$. "Pour exprimer la substance de son propos, l'écrivain se

27. AUDET, Louis-Philippe, Le frère Marie-Victorin éducateur : ses idées pédagogiques, 16 planches et bibliographie par Marcelle GAUVREAU, Québec, Éditions de l'Érable, 1942.

28. GAUVREAU, Marcelle, Le président de l'ACFAS pour 1937-1938 : le frère Marie-Victorin, biographie et bibliographie, Montréal, s.n., 1938.

29. MAXINE, Les trois fées au bois d'épinette. La nature, ses règnes, ses merveilles, préface d'Albert LÉVESQUE, Montréal, Albert Lévesque, 1936.

30. BERNARD, Harry, L'ABC du petit naturaliste canadien, Montréal, Librairie Granger, 1936.

31. MAXINE, Les trois fées..., op. cit., p. 7.

32. Ibid., p. 9. 
sert du procédé du miroir ou du " texte dans le texte ", si bien que chaque fée révèle un livre d'or aux enfants afin d'exposer les éléments relatifs au domaine qu'elle personnifie. Par exemple, la partie consacrée au voyage de Pierrot dans le royaume du règne animal, en compagnie de la fée Vigueur, se divise en quatre sections correspondant à chaque ordre inauguré par la formule $\mathrm{ABC}$, formule qui atteste nommément du niveau d'apprentissage : on y traite des oiseaux, des mammifères, des poissons et des insectes. Chaque section comporte des entrées qui respectent l'ordre alphabétique. Qui plus est, chaque exposé renferme une définition concrète et vivante, exempte de métalangage et agrémentée de détails pouvant susciter d'emblée l'attention, ce qui confère une portée pragmatique à l'énoncé descriptif. Selon cette perspective, on y interpelle hardiment le jeune lecteur. À la rubrique "Pic ", on peut lire :

"Regarde ce tronc d'arbre... vois-tu cet oiseau qui est en train de le marteler à coups de bec? C'est un PIC [sic]! Il mange les petits insectes qu'il trouve dans l'écorce. Le pic est très joli, avec son plumage tacheté, mais quand tu l'entendras faire toc... toc... sur le bois de l'écorce, tu peux être certain qu'il annonce une averse prochaine ${ }^{33}$."

La section portant sur les fleurs champêtres, les arbres et les plantes abreuve la jeune Lili qui a amorcé avec la fée Croissance un voyage à travers les somptueux coloris du règne végétal. Cette partie contient à son tour de nombreux appels au destinataire. À l'exemple de la première partie, la seconde qui parle de la végétation a le mérite de ne pas sombrer dans les termes techniques qui rebutent les enfants :

«Le bouleau est ce bel arbre si précieux pour son ombrage, son bois et son écorce blanche. C'est avec cette écorce que l'on fabrique les canots qui filent si rapidement sur les rivières et les lacs. [...] B c'est encore le BLEUET $[s i c]$ ce fruit que tu aimes à cueillir, jolie baie bleue et savoureuse qui croît avec abondance sur de petits arbrisseaux sauvages ${ }^{34}$ ".

Contrairement au poète Chapman qui utilise à tort l'expression « lis immaculé ", Maxine commente l'entrée " lis " sans verser dans le lieu commun :

"Le superbe LIS [sic] blanc croît dans les jardins et les serres, mais il y a dans nos champs de beaux LIS couleur orange qui fleurissent en juillet parmi l'avoine et le foin ${ }^{35}$."

Quant aux termes " glaïeul " et " ajonc ", ils ne figurent pas au sein de l'exposé ayant trait aux fleurs champêtres et aux plantes vivaces. Le lecteur avisé peut constater que l'écrivain a consulté des manuels d'histoire naturelle avant de rédiger sa fable. La partie consacrée au royaume de la fée Richesse s'articule selon les mêmes paramètres, bien qu'elle soit moins volumineuse que les deux premières. La narratrice fée prend soin de préciser quels sont les minéraux que l'on trouve au Canada :

33. Ibid., p. 26

34. Ibid., p. 58-59.

35. Ibid., p. 53. 
" N c'est le NICKEL [sic] un métal fort utile dont le Canada possède des mines importantes."

«L'ÉMERAUDE [sic] est une pierre précieuse de belle couleur verte que l'on ne trouve pas dans notre pays ${ }^{36}$."

À divers degrés, l'ouvrage de Maxine fait écho aux enseignements du frère Marie-Victorin et à la ferveur qui animait ce dernier quand il décrivait les paysages et l'habitat de la vallée laurentienne.

L'avènement de la presse enfantine dans les années 1920 a joué un rôle déterminant dans l'histoire de la littérature de jeunesse canadienne-française, en général, et dans le développement des écrits de vulgarisation scientifique, en particulier. Auteur des Fées de la terre canadienne ${ }^{37}$, un texte lui-même traduit de l'anglais, Maxine a publié plusieurs œuvres à caractère historique qu'elle destinait à un jeune public. À l'instar de Maxine, Marie-Claire Daveluy fait figure de pionnière puisqu'elle a marqué à son tour l'édition pour la jeunesse. Rappelons que le premier ouvrage écrit pour les enfants a paru en 1923 sous forme de feuilleton dans L'Oiseau bleu, la première revue à l'intention d'un jeune public, publiée en français au Canada, sous les auspices de la Société Saint-Jean Baptiste qui a œuvré en faveur de l'éducation populaire et de l'enfance. Il s'agit des Aventures de Perrine et de Charlot $^{38}$ de Marie-Claire Daveluy, un roman d'aventures dont les principaux personnages sont des enfants et dont l'intrigue se déroule aux premiers temps de la colonie. Comme le note Jeanne Saint-Pierre dans "Ce que lisent les jeunes au Canada", "le mouvement commencé, d'autres écrivains, pionniers eux aussi, se mirent à écrire spécialement pour les enfants, la plupart de ces auteurs s'efforceront de donner à leurs travaux un caractère historique plus ou moins prononcé ${ }^{39}$ ". À partir de janvier 1932, L'Oiseau bleu ouvre ses pages à des domaines plus scientifiques : zoologie, botanique, minéralogie, à la suite de la création des Cercles des jeunes naturalistes, si chers au frère Marie-Victorin. Mis sur pied en 1931, ces cercles privilégient l'étude sur le terrain, l'observation en pleine nature, comme le prescrit l'enseignement de l'homme de science. De plus, on publie régulièrement jusqu'en 1940 des articles de botanique sous la direction de Marcelle Gauvreau, qui est en charge de l'éducation des jeunes de 4 à 7 ans en matière de botanique, dans le cadre des activités de "L'école de l'éveil " rattachée au Jardin botanique de Montréal. Comme l'indique Françoise Lepage dans son Histoire de la littérature pour la jeunesse, "cette

36. Ibid., p. 73 et 71 .

37. MAXINE, Fées de la terre canadienne, traduction de Unknown Fairies of Canada, Montréal, éd. de l'Action canadienne-française, 1928.

38. Daveluy, Marie-Claire Daveluy, Les aventures de Perrine et de Charlot, Montréal, Bibliothèque de l'Action française, 1923; d'abord publiée dans L'Oiseau bleu (janvier 1921décembre 1922). "Ce premier roman se prolongera en fait en un cycle romanesque qui sera publié plus tard en six volumes (1923-1940). " (LEPAGE, Françoise, op. cit., p. 118)

39. SAint-Pierre, Jeanne, "Ce que lisent les jeunes au Canada ", Le livre de l'année, Montréal, Grolier, 1952, p. 169. 
ouverture sur les sciences dénote un progrès vers la modernisation des matières enseignées ${ }^{40}$ ", si bien que les "petites sciences " ne tardent pas à changer de statut. Au sein d'une synthèse historique des sciences au Québec, Luc Chartrand, Raymond Duchesne et Yves Gingras soutiennent que « Marie-Victorin assure cette liaison essentielle entre les intellectuels et l'ensemble de la société [durant la période de l'entre-deux-guerres] ${ }^{41}$ ". Les auteurs de l'Histoire des sciences au Québec mentionnent que « le charisme du scientifique est à la source de l'influence qu'il exerce sur le mouvement intellectuel durant cette période ". Dans le prolongement de ces affirmations, il est justifié d'avancer l'hypothèse que le frère Marie-Victorin a donné un élan décisif à la production littéraire pour la jeunesse, orientée vers la vulgarisation des sciences. Une analyse détaillée de l'ensemble des textes parus dans les années trente et quarante permettrait bien entendu de confirmer pareille hypothèse.

Force est d'admettre que l'intérêt des écrivains pour la vulgarisation scientifique s'accroît considérablement à une époque où l'on clame « la science pour tous ". Comme en fait état déjà une partie de la production des années 1930 et 1940 , des auteurs entreprennent de populariser les sciences au sein de textes qu'ils destinent à la jeunesse. Le rôle majeur qu'a joué le frère Marie-Victorin à travers la diffusion de son œuvre scientifique puis à l'intérieur d'un mouvement de jeunesse, soit le Cercle des jeunes naturalistes - qui accueillait d'ailleurs beaucoup plus de filles que de garçons $^{42}$-, aura eu un impact déterminant sur la société québécoise et sur les écrivains soucieux de rendre la science accessible aux jeunes et de populariser les discours savants au moyen de la fiction et du documentaire.

40. LEPAGE, Françoise, op. cit., p. 116.

41. CharTRAND, Luc, DuchESNE, Raymond, et GingRas, Yves, Histoire des sciences au Québec, Montréal, Boréal, 1987, p. 261.

42. Pierrick MALISSARD arrive à cette conclusion à partir d'une analyse détaillée d'une partie des archives des Cercles des jeunes naturalistes. "Les cercles des jeunes naturalistes. Ampleur et nature du mouvement, 1931-1971 ", Revue d'histoire de l'Amérique française, vol. $50, \mathrm{n}^{\circ} 1$, été 1996 , p. 19. 


\section{RÉSUMÉ}

Cet article examine l'influence des écrits du frère Marie-Victorin (18851944) sur des œuvres littéraires pour la jeunesse. Dans le domaine des sciences naturelles, les travaux de ce dernier ont inspiré en effet nombre d'écrivains durant les années 1930 et 1940 au Québec. Nous y abordons dans un premier temps les écrits du botaniste afin de décrire son influence à la fois dans le milieu scientifique et dans le champ littéraire. Dans un deuxième temps, nous proposons une lecture de quelques textes pour la jeunesse des années 1930 et 1940, dont le code est dicté en grande partie par l'œuvre de l'homme de science. Plus précisément, Les trois fées du bois d'épinette (1936) de l'écrivain Maxine est soumis à l'analyse. L'étude montre que plusieurs femmes, à la suite du frère Marie-Victorin, ont choisi de rendre la science accessible aux jeunes et de populariser les discours savants au moyen de la fiction et du documentaire.

\section{ABSTRACT}

This article examines the influence of the writings of Brother Marie-Victorin (1885-1944) on young people's literature. Marie-Victorin's work in the field of the natural sciences, in fact, inspired a number of Québec writers during the decade between 1930 and 1940. We begin by describing the influence of the botanist's writings in the fields of both science and literature. Next, we propose to examine a few texts for young people in Québec between 1930 and 1940, where the code was largely dictated by the work of this man of science. We focus, more specifically, on the text Les trois fées du bois d'épinette [The Three Fairies in the Spruce Wood] (1936) by the writer Maxine. This article shows that after Brother Marie-Victorin, a number of women chose to make science accessible to young people and popularize scholarly discourse by means of fiction and documentaries. 
Achevé d'imprimer

sur les presses du service reprographique de l'université Rennes 2 Haute-Bretagne en décembre 2003 


\section{QUELQUES RÈGLES DE PRÉSENTATION DES TEXTES À L’USAGE DES AUTEURS}

1 - Indiquer sous le titre de l'article la qualité et l'adresse institutionnelle de l'auteur Ex. : Xxxx Xxxxxx, maître de conférences en histoire contemporaine, université de X., groupe de recherches de rattachement.

2 - Remplacer le plus possible les majuscules (sauf pour les lettres initiales des noms propres) par des petites capitales. Ceci dans les titres, les sigles, les noms d'auteurs...

Accentuer les majuscules.

3 - Abréviations : en user le moins possible

Pas d'abréviations pour le nom des revues, surtout pour les revues locales qui ne sont connues que des locaux.

Pour les dépôts d'archives :

- ne pas mettre ADIV ou AD 35 mais : Arch. dép. d'llle-et-Vilaine, du Morbihan...;

- préférer Arch. nat. à AN;

- mettre les noms des bibliothèques en entier.

Développer les sigles entre parenthèses lors de leur première occurrence.

4 - Références bibliographiques

- mettre toujours et partout les prénoms et les noms (ne pas abréger le prénom par une initiale);

- utiliser les petites capitales pour le nom propre et non les majuscules, utiliser les minuscules pour le prénom; les séparer par des virgules.

Nom, Prénom, Titre de l'ouvrage en italique, Lieu d'édition, éditeur, " collection " (éventuellement), année, pages.

Une bibliographie peut être donnée en fin d'article si cela est nécessaire.

5 - Forme des notes

Préférer la forme traditionnelle (référence biblio. développée lors de sa première occurrence, abrégée ensuite) à la forme anglo-saxonne (nom, date, page).

\section{6 - Titres et sous-titres}

Il est préférable de s'en tenir à deux niveaux. Mais si cela s'avère nécessaire afin de ne pas obtenir de trop longues pages trop compactes, un troisième niveau d'intertitres peut être utilisé.

7 - Cartes et documents photographiques

- Les cartes au trait devront être d'une EXCELLENTE QUALITÉ GRAPHIQUE sans collage hasardeux ni légende manuscrite.

- Il est toujours préférable de FOURNIR LES ORIGINAUX, sinon, un fichier informatique au format .EPS pour les cartes et plans ou .TIF pour les photographies. En aucun cas un ficher .JPG, de résolution trop basse.

8 - Autres questions non évoquées ici...

consulter la rédaction à l'adresse ci-dessous.

9 - Donnez une adresse mail et téléphone où l'on puisse vous joindre facilement au moment où l'on fait la mise en page de votre article.

\section{Contact}

Annie Antorne, Secrétaire de l'Association pour la publication des $A B P O$ université de Rennes 2, Département Histoire,

Place du Doyen-Henri-Le-Moal - 35043 RenNEs Cedex annie.antoine@uhb.fr

Les manuscrits sont à envoyer à l'adresse ci-dessus 